\title{
Preoperative localization of sentinel lymph nodes using percutaneous contrast-enhanced ultrasonography in patients with breast cancer
}

\author{
Yi-Lin Xu", Xue-Jing Liu", Ying Zhu, Hong Lu \\ Department of Breast Imaging, Tianjin Medical University Cancer Institute and Hospital, National Clinical Research Center of Cancer, Key \\ Laboratory of Cancer Prevention and Therapy of Tianjin (Ministry of Education), Key Laboratory of Breast Cancer Prevention and Therapy of \\ Education Ministry, Tianjin, China \\ Contributions: (I) Conception and design: YL Xu; (II) Administrative support: H Lu; (III) Provision of study materials or patients: YL Xu, XJ Liu, Y \\ Zhu; (IV) Collection and assembly of data: YL Xu; (V) Data analysis and interpretation: YL Xu, XJ Liu; (VI) Manuscript writing: All authors; (VII) \\ Final approval of manuscript: All authors. \\ \#These authors contributed equally to this work as co-first authors. \\ Correspondence to: Hong Lu. Department of Breast Imaging, Tianjin Medical University Cancer Institute and Hospital, National Clinical Research \\ Center of Cancer, Key Laboratory of Cancer Prevention and Therapy of Tianjin (Ministry of Education), Key Laboratory of Breast Cancer \\ Prevention and Therapy of Education Ministry, Tianjin 300060, China. Email: honglu@tmu.edu.cn.
}

Background: This study aimed to investigate the feasibility of preoperative identification of sentinel lymph
nodes (SLNs) by contrast-enhanced ultrasound (CEUS) for patients with breast cancer.
Methods: The patients with T1-T2N0M0 breast cancer who were scheduled for primary surgical
treatment were recruited. All the patients had received a periareolar intradermal injection of an ultrasonic
contrast agent (SonoVue, Bracco, Milan, Italy) followed by an ultrasound to identify contrast-enhanced
SLNs. A guidewire was deployed to localize the SLN. Methylene blue stain was used to help trace SLNs
during the operation. The identification rate and accuracy rate were recorded. The number of SLNs labeled
by two methods was counted and compared using Wilcoxon testing.

Results: A total of 366 SLNs were detected in 72 patients by methylene blue intraoperatively, with a median of 5 lymph nodes [interquartile range (IQR), 4-6] per patient. A total of 95 SLNs were detected in 63 patients $(87.5 \%)$ by CEUS, with a median of 1 lymph node (IQR, 1-2) per patient. The number of SLNs detected by CEUS was significantly less than that labeled by the methylene blue staining method $(Z=-7.362$, $\mathrm{P}=0000)$. Pathology confirmed 12 single metastases in all the lymph nodes examined, 10 of which were the only lymph node identified by CEUS.

Conclusions: Periareolar intradermal injection of an ultrasonic contrast agent was an effective and convenient supplementary to localize SLNs. The technique was expected to improve the accuracy of axillary staging with minor surgical trauma and postoperative complications.

Keywords: Breast diseases; contrast-enhanced ultrasound (CEUS); sentinel lymph node (SLN)

Submitted Dec 07, 2021. Accepted for publication Feb 18, 2022.

doi: 10.21037 /gs-22-10

View this article at: https://dx.doi.org/10.21037/gs-22-10

\section{Introduction}

Breast cancer has surpassed lung cancer as the most commonly diagnosed cancer and the deadliest cancer affecting women worldwide, with an estimated 2.3 million new cases $(11.7 \%$ of the total new cases) in 2020 (1). In patients with a positive sentinel lymph node biopsy, the conventional approach is axillary lymph node dissection (ALND). But only a few patients would develop 
metastases in the remaining lymph nodes, ALND may be overtreatment for early-stage breast cancer patients with low axillary tumor burden (2). Reducing patient injuries is an important challenge in precision breast surgery. The sentinel lymph node (SLN) is the hypothetical first lymph node receiving lymphatic drainage from the primary tumor, which can accurately determine the status of axillary nodes. In patients with early breast cancer, SLN biopsy (SLNB) has gradually become an important alternative to ALND, as traditional ALND carries a greater risk of complications, such as lateral upper limb pain, numbness, edema, and movement disorders (2-7). Patients with no clinical involvement of the axilla should have SLNB performed routinely, and when the sentinel node is disease-free, no additional lymph node surgery is required.

Methods for the precise localization of SLNs have caused close clinical attention. The most commonly used SLN tracers for patients with breast cancer include blue-staining and nuclear tracers. To improve the success rate of SLNB and to reduce the false-negative rate, a combination of blue stain and nuclear tracers is recommended first $(8,9)$. The methylene blue method has been widely used due to its simplicity and low cost. However, due to the small molecular weight of blue staining, it is more prone to entering secondary lymphatic vessels or lymph nodes, resulting in excessive blue staining of non-sentinel lymph nodes and more postoperative complications. Allergic reactions also limit the application of the methylene blue method (10). Radioactive nuclides have a higher sensitivity to SLNs (11), but the small colloidal particles that enable the visualization of SLNs also reach other lymph nodes. Previous Research has shown that the lymph nodes with the most radioactive node may not always be the SLN (12). The clinical application of radionuclide is also limited by its cost and radiation damage (10). The fluorescent tracer technique can be used as an optional technique. The value of nanocarbon as a tracer needs to be further confirmed.

Ultrasonography is the most frequently used preoperative technique to determine axillary lymph node status. But conventional gray-scale ultrasonography has difficulty in identifying which lymph node is the sentinel node, especially in patients with no or only micrometastases in axillary lymph nodes. Although preoperative axillary gray-scale ultrasonography and fine-needle aspiration cytology for suspicious lymph nodes can detect lymph node metastases, the sensitivity is only 30.6-62.9\% (13). Therefore, some researchers started looking for a novel sentinel lymph node (SLN) tracking approach.

Goldberg (14) established a porcine melanoma model and found that SLNs were exactly identified in $90 \%$ of animal models injected with the contrast agent into the lymphatic vessels around the tumor. The value of contrastenhanced ultrasound in human breast cancer SLN was first reported in 2006 by Omoto et al. (15). The results showed that CEUS is an effective method for identifying SLN in human breast cancer. In the last decade, several clinical studies have investigated the use of contrast-enhanced ultrasonography to identify sentinel lymph nodes in earlystage breast cancer and their utility in sentinel lymph node biopsy $(8,16-23)$. CEUS has been shown in several trials to improve SLN detection and reduce SLNB surgical trauma. However, some have revealed that SLNB guided by CEUS alone might have a false negative result and limited diagnostic accuracy $(8,16-23)$. Our purpose in this paper was to investigate the feasibility of preoperative identification of SLNs by CEUS for patients with early breast cancer.

We present the following article in accordance with the STARD reporting checklist (available at https:// gs.amegroups.com/article/view/10.21037/gs-22-10/rc).

\section{Methods}

From December 2016 to June 2018, consecutive patients with T1-T2N0M0 breast cancer who were scheduled for primary surgical treatment in our hospital were recruited into the study (Figure 1). The inclusion criteria were as follows: (I) patients with no history of chemotherapy, radiotherapy, or axillary surgery; and (II) patients with single breast lesions (multiple breast lesions should be excluded by mammography, ultrasound, or MR before the operation). The exclusion criteria were as follows: (I) patients who were pregnant or lactating; (II) patients with inflammatory breast cancer; and (III) patients who were allergic to ultrasound contrast agents.

All procedures performed in this study were in accordance with the Declaration of Helsinki (as revised in 2013). The study was approved by Institutional Review Board of Tianjin Medical University Cancer Institute and Hospital (No. bc2018015). Individual consent for this retrospective analysis was waived.

All sonographic examinations were performed with either a GE Logiq E9 (GE Healthcare, Chicago, IL, USA) or a Philips EPIQ5 color Doppler (Philips, Amsterdam, the Netherlands) ultrasound system. The grayscale ultrasounds 


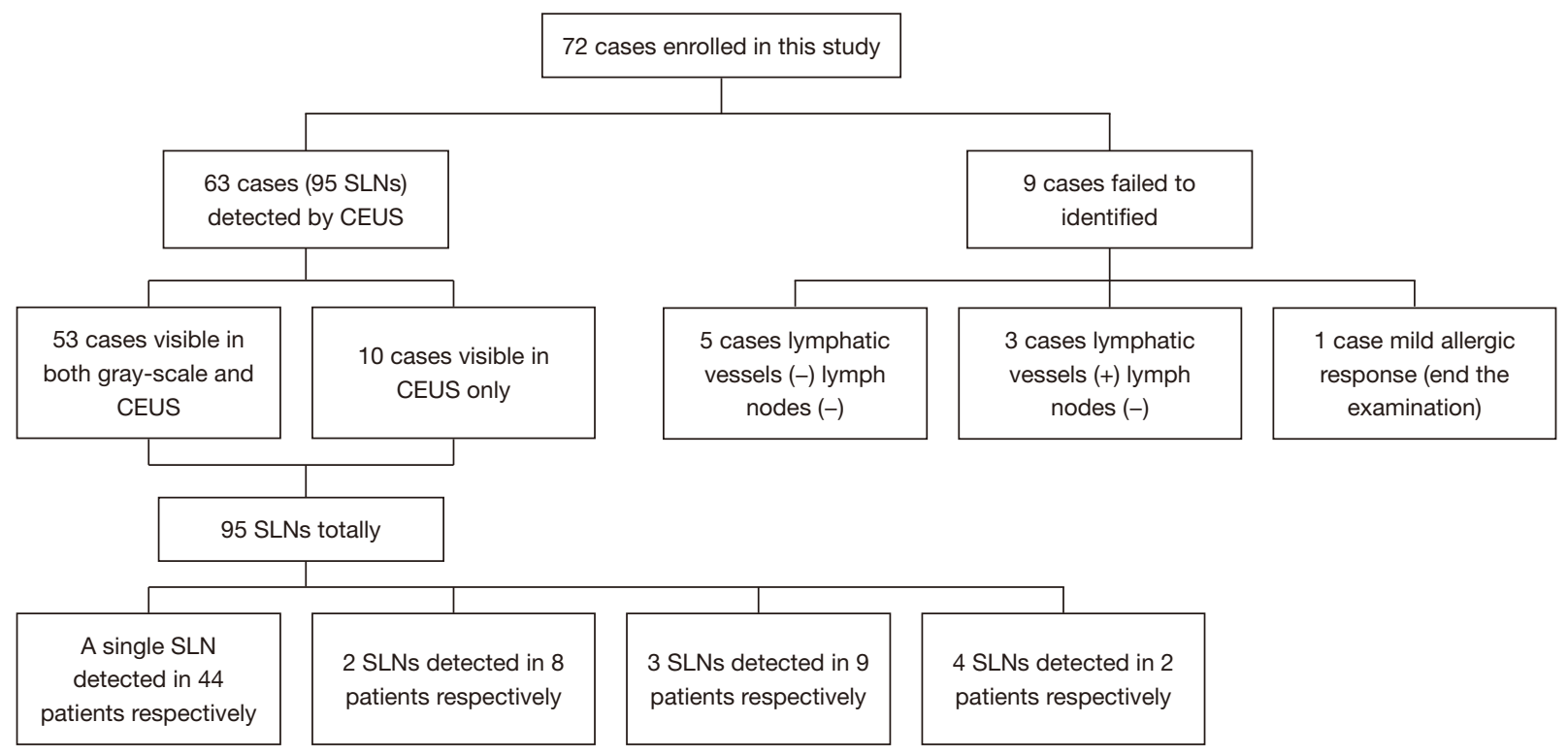

Figure 1 Flowchart of this paper. CEUS, contrast-enhanced ultrasound; SLN, sentinel lymph node.

were performed with either ML6-15 (GE Healthcare) or EL18-4 (Philips) linear array probes with a frequency of 10-12.0 MHz. For CEUS, a 9L (GE Healthcare) or an EL18-4 (Philips) linear array probe was used with a probe frequency of 7-12 MHz and a low mechanical index (MI) of $0.08-0.22$. The procedure was performed by two senior ultrasound doctors with more than 5 years of experience.

The patient was placed in the supine position with the upper limbs lifted and abducted behind the occiput to fully expose the axilla. A routine ultrasound was performed to assess the breast mass and axilla and preliminarily determine the possible location of SLNs. After routine disinfection of the affected breast and axillary skin, $1 \%$ lidocaine was used to subcutaneously infiltrate the areola area for local anesthesia. An Ultrasound contrast agent suspension (between 0.2 and $0.5 \mathrm{~mL}$ ) was injected intradermally on the skin of the areola in the upper outer quadrant. After the injection, the injection site was gently massaged to accelerate the entry of the contrast agent into the lymphatic vessels. Using the contrast mode, the first enhanced lymph node tracked from the injection site to the enhanced lymphatic vessel was considered as the SLN and marked on the body surface. In most cases, it took about $20 \mathrm{~s}$ from injection to development and could last up to $3 \mathrm{~min}(8)$. Repeated areola massage could usually redevelop SLN without a second injection. Preoperative wire-localization guided by ultrasound and body surface marking was performed in the SLN identified (Figure 2). The number, location, both distances from the body surface and the pectoral muscles of the SLNs were recorded. If either enhanced lymph nodes or lymphatic filling were not shown obviously after three times injection repeated, the case was considered a failure.

Immediately after induction of anesthesia, patients received a $2 \mathrm{~mL}$ subdermal injection of methylene blue in the periareolar upper outer quadrant region. The blue dyecontaining lymph nodes or blue-stained lymphatic vessels leading to lymph nodes were considered as SLNs. All guidewire-containing lymph nodes, all blue-stained lymphatic vessels, and lymph nodes were completely removed and submitted for examination using the SLNB criteria of the University of Louisville in the United States (24).

\section{Statistical analysis}

In this paper commercially available statistical software (IBM SPSS Statistics version 20) was used for data analysis. The normality of the distribution was assessed using the Shapiro test and histogram visualization. Data following non-normal distribution were expressed as a median (interquartile range). Wilcoxon testing was used to assess for differences of SLNs numbers detected by percutaneous CEUS and blue dye. In comparison with the blue dye results, the accuracy of percutaneous CEUS localization of SLN was calculated. $\mathrm{P}$ values $<0.05$ were considered to indicate statistical significance in all analyses. 

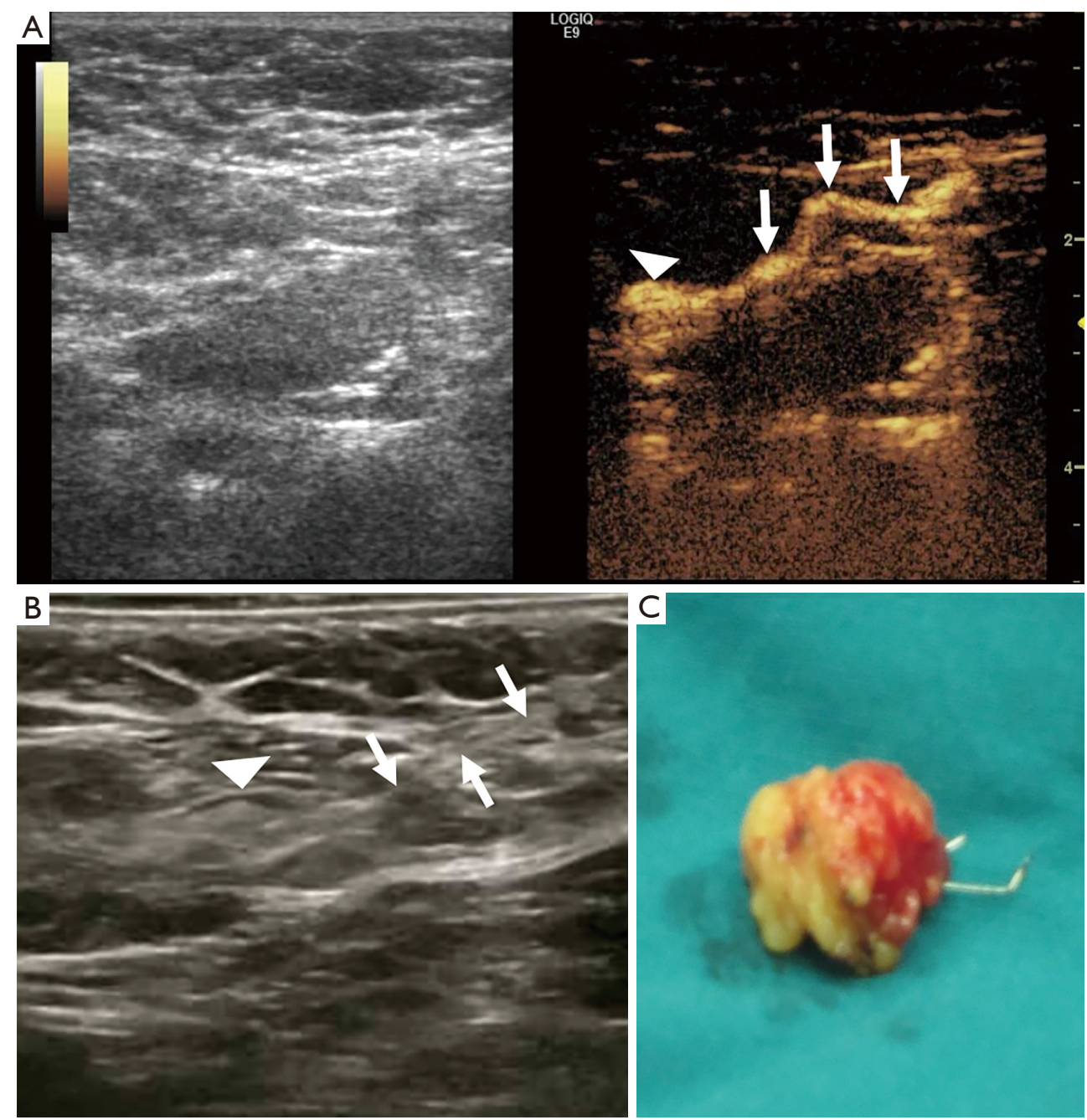

Figure 2 Female, 57 years old, with left breast cancer. (A) The first developed lymph node (arrowhead) detected by the developed lymphatic vessel (arrow) is identified as the SLN; (B) SLN positioned (arrowhead) by wire (arrow) under ultrasound guidance; (C) lymph nodes located during the operation. SLN, sentinel lymph node.

\section{Results}

A total of 72 patients with stage T1-T2N0M0 breast cancer were enrolled, ranging in age from 28 to 68 years old, with a median age of 53 years old. The primary breast cancer lesions included 35 cases in the upper-outer quadrant, 12 in the upper-middle quadrant, 7 in the middle outer quadrant, 6 in the lower outer quadrant, 1 in the lower quadrant, 2 in the inner lower quadrant, 4 in the inner side, and 5 in the upper inner quadrant.

The methylene blue method detected a total of 366 SLNs in 72 patients. The median number of SLNs was 5 (IQR, 4-6) per patient. Pathology confirmed that a total of 12 lymph nodes in 12 patients were metastatic lymph nodes, and the remaining lymph nodes were all negative. A total of 95 SLNs in 63 patients were detected by intradermal injection of an ultrasound contrast agent in the areola area. The overall detection rate was $87.5 \%(63 / 72)$, with a median number of SLNs being 1 (IQR, 1-2) per patient, which was less than the methylene blue group [5 (IQR, 4-6)] and the difference was statistically significant $(\mathrm{Z}=-7.362$, $\mathrm{P}=0000$, Table 1). In 53 patients, SLNs were visible by both grayscale ultrasound and contrast-enhanced ultrasound. In 10 patients, SLNs were recognized only after CEUS and only then seen as a typical negative lymph node with a thin cortex on grayscale imaging (Figure 3). In the CEUS group, a single SLN was detected respectively in 44 patients 
Table 1 The number of SLNs in the CEUS group and methylene blue group

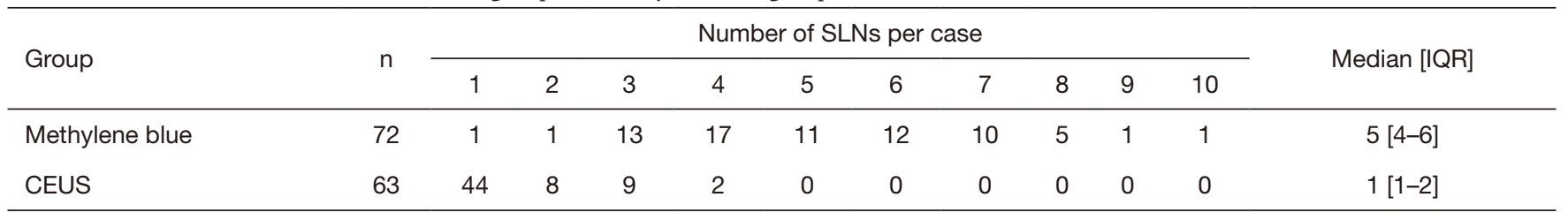

$\mathrm{Z}=-7.362$, $\mathrm{P}=0000$. SLNs, sentinel lymph nodes; IQR, interquartile range; CEUS, contrast-enhanced ultrasound.

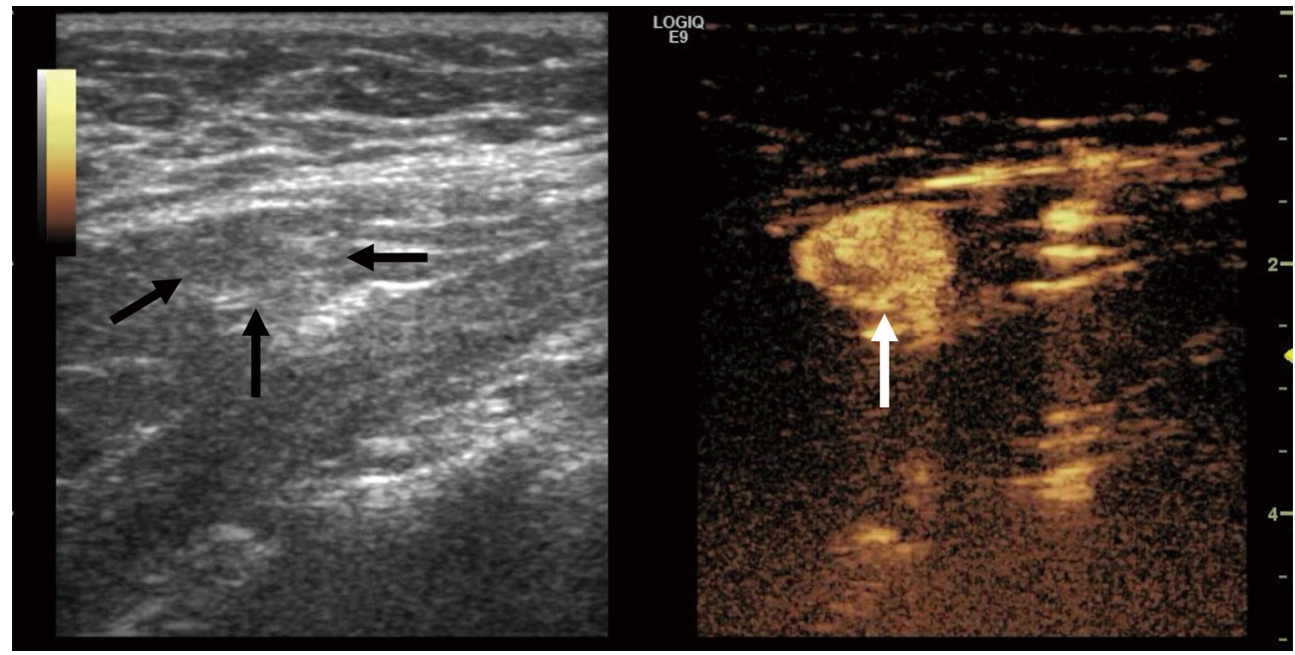

Figure 3 Real-time double-frame ultrasound contrast image. Lymph nodes with significant enhancement (white arrow). Before CEUS, the lymph node and the surrounding tissues had almost no echo intensity difference, which was difficult to identify. When the 2 images were compared with each other during ultrasound enhancement, the position of the SLN could be confirmed (black arrows). SLN, sentinel lymph node; CEUS, contrast-enhanced ultrasound.

(66.7\%). Among the other 19 patients, 4 lymph nodes were detected in 2 patients, 3 nodes were identified in 9 patients, and 2 nodes were detected in 8 patients. The 12 metastatic lymph nodes confirmed by histopathology were all single metastases; 10 of these were the single lymph nodes located by contrast-enhanced ultrasound. In the other 2 patients, 3 and 4 SLNs were detected, respectively. In 9 patients, the procedure failed. In 5 patients, lymphatic vessels and lymph nodes were not detected. In 3 patients, the lymphatic vessels were visualized but the lymph nodes were not visualized. An allergic response (a slight itching in the injection site) occurred in 1 patient; no other adverse events occurred.

\section{Discussion}

Ultrasound contrast agents are composed of phospholipidencapsulated sulfur hexafluoride microbubbles without protein components, and thus allergic reactions are relatively rare (25). The lymphatic capillaries are about $10-50 \mu \mathrm{m}$ in diameter, composed of a single layer of endothelial cells overlapping each other. The function of the joint is similar to a valve, with a width of 10-25 $\mu \mathrm{m}$ when open, allowing small particles to pass through. The diameter of the SonoVue microbubble is about $1.0-10.0 \mu \mathrm{m}$, with an average diameter of $2.5 \mu \mathrm{m}$. Therefore, the microbubbles can smoothly enter the lymphatic drainage pathway, drain from the lymphatic vessels to the SLN, and enhance the reflected signal by increasing the backscatter of the ultrasound to visualize the draining lymphatic vessels and lymph nodes. Transvenous injection and subcutaneous injection are two main injection strategies for ultrasonography tracking SLN, and the ideal injection location is still up for debate. The detection rate of transdermal lymph node ultrasonography tracing SLN was $90.10 \%$, which was much greater than that of transvenous injection $(29.36 \%)$, according to Yang's study (26). Furthermore, the results revealed that subcutaneous ultrasonography contrast tracing of SLN had greater sensitivity, accuracy, and specificity than transvenous 


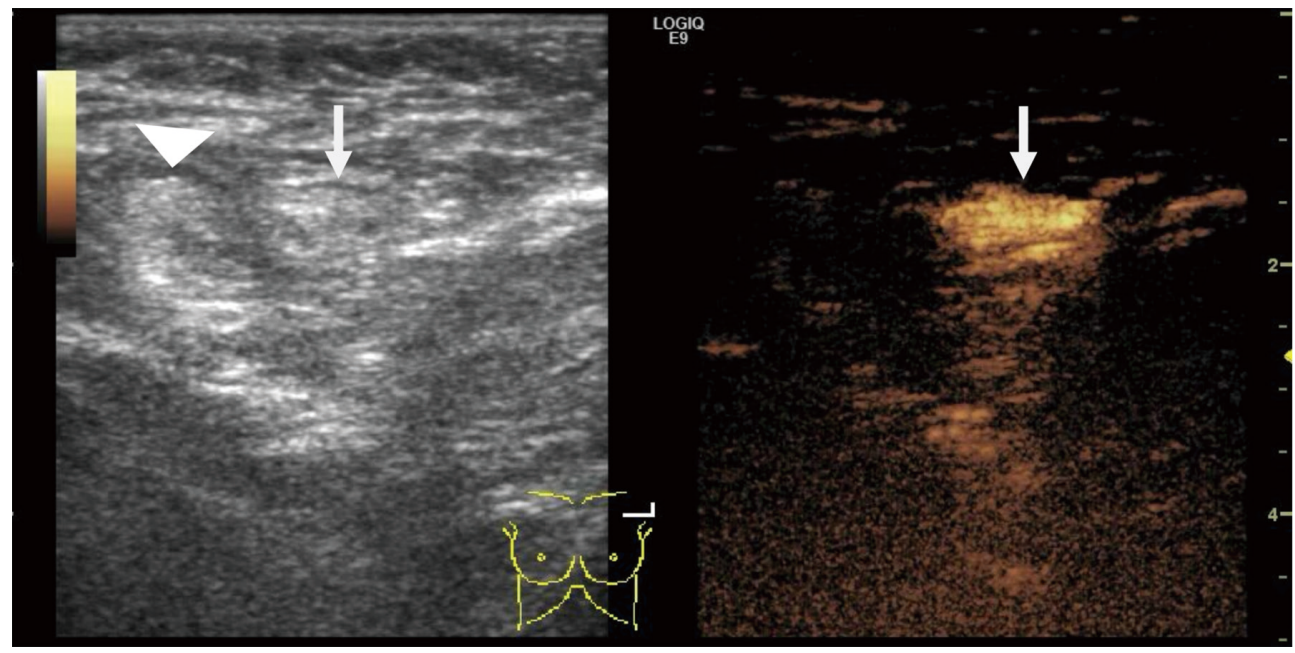

Figure 4 Real-time double-frame ultrasound contrast image. The first lymph node (arrow) that developed along the draining lymphatic vessel was identified as the SLN, and the adjacent lymph node (arrowhead) did not develop, indicating that the contrast agent microbubbles did not easily flow out after entering the SLN. SLN, sentinel lymph node.

injection. Comparing the 2 methods, transdermal injection had the following advantages. (I) Breast lymphatic fluid drained from the capillary lymphatic vessels under the areola and then to the ipsilateral axillary lymph nodes. (II) The subcutaneous lymphatic system under the areola was rich and lax in structure, and when injected subcutaneously, the contrast agent could quickly enter the lymphatic vessels and clearly showed the SLN along with the enhancer. (III) Transvenous injection of contrast medium could clearly show the microcirculatory perfusion of lymph nodes through the blood circulation system. The detection rate of SLN was significantly reduced because the travel and enhancement of lymphatic vessels could not be shown $(26,27)$.

In this study, percutaneous injection of an ultrasound contrast agent was used. A proportion of $87.5 \%(63 / 72)$ of the patients were identified by the CEUS method. Only 1 SLN was detected respectively in the majority of patients $(66.7 \%)$, with a median number of 1 . The CEUS detection rate was similar to the results of previous studies $(8,16-23,26,27)$. Theoretically, SonoVue microbubble particles are larger than methylene blue or nuclide. Once introduced into the lymph node the microbubbles are ingested by the reticuloendothelial cells, rather than passing through the lymph node and entering the next node $(20,21)$ (Figure 4), and thus it will not enhance the adjacent lymph nodes in a large range and can be maintained for a long time. In this study, 366 SLNs in 72 patients were detected by the methylene blue method, with a median number of SLNs
5 (IQR, 4-6) per patient. A significantly smaller number of SLNs was detected by the CEUS method $(\mathrm{P}<0.01)$. A meta-analysis (10) showed that although the number of CESLNs (sentinel lymph nodes identified by contrast-enhanced ultrasound) was significantly less than that of non-CE-SLNs (sentinel lymph nodes identified without contrast-enhanced ultrasound). However, the risk of positive CE-SLNs was six times higher than non-CE-SLNs. Similarly, 12 SLNs in 12 cases were metastatic in this study. In 10 of these, the SLN was the only lymph node located by CEUS. It's suggested that CEUS had higher accuracy than traditional methods, and CE-SLN might be a more accurate sentinel lymph node. As a result, the requirement for sentinel lymph node detection as well as the trauma of axillary surgery will be reduced. In 10 cases SLNs were seen on grey-scale ultrasound, only after CEUS. The lymphatic vessels were visualized by CEUS (Figure 3). Without CEUS, the grayscale ultrasound might miss the SLN, which suggested that ultrasound contrast could improve the sensitivity and accuracy of SLN detection. Unlike conventional ultrasound, CEUS could allow for targeted ultrasound-guided biopsy of SLNs in patients with breast cancer preoperatively. Sever's research indicated that the patients proven SLN metastases later could skip the unnecessary SLN excision biopsy and go straight to ALN dissection at the time of breast surgery (8).

Recent studies indicated that in early-stage breast cancer with limited sentinel node metastasis, axillary lymph node dissection may not be superior to sentinel lymph node 
dissection (SLND) (2-7). Both the IBCSG 23-01 and AATRM 048/13/2000 trails found that ALND may be safely avoided without reducing disease-free survival in patients with SLND micrometastasis. SLND/Radiotherapy management was recommended for early breast cancer patients with $<3$ positive SLNs with either micrometastasis or macrometastasis $(6,7)$. The excision of the CE-SLN might be adequate, and there was a potential benefit of limiting the scope of surgery and injury (10). The enhanced pattern had certain significance in the diagnosis of SLN metastasis. The absence of enhancement in metastatic SLN was attributable to tumor metastasis or destruction of normal tissue (16).

A total of 9 cases failed and in the CEUS method. In 1 patient, the inspection was terminated due to allergic response. In another 3 patients, the lymphatic vessels were visualized but the lymph nodes were not visualized. In the other 5 patients, the lymphatic vessels and lymph nodes were not detected. Considering previous research, the possible reasons for this are analyzed as follows. First, the tumor might have invaded the local lymphatic vessels and formed lymphatic cancer thrombi, which might have caused the tracer to fail to pass $(10,23)$. Secondly, about $3 \%$ of breast lymph nodes drained to the internal mammary lymphatic chain, so the first metastasis of some breast cancers might be located in the internal mammary area. If the injection position of the contrast agent could be adjusted to the inside of the areola, these internal mammary lymph nodes might be detected. Thirdly, the patient might have experienced feelings of stress and anxiety that made it impossible to relax, which might have affected lymphatic drainage.

Another advantage of CEUS was the ability to image SLNs in real-time. It was possible to see a dynamic process of SLN amplification from the afferent lymphatic vessel to the efferent vessel $(16,19)$. The most common marking methods for SLNs were skin labeling and guidewire localization. Other labeling methods include titanium clip, iodine 125 particle implantation, ultrasoundguided injection of fluorescent dye, and so on $(10,17,23)$. Since SLNs might not be isolated from non-SLNs in some cases, skin labeling alone might lead to inaccurate results. The titanium clips were too tiny to be seen or felt intraoperatively, which might make clinical application problematic, whereas a guidewire would be more effective (23). This paper employed wire-localization combing skin marking based on the scenario. Once the SLN were recognized by CEUS, the guidewire deployment and skin recognition would be completed before surgery.
This process eliminated the time of intraoperative blue staining, which potentially reduced the risk of surgery. Skin labels were pretty intuitive for surgeons $(8,16)$.

This study had several limitations. First of all, the number of patient samples in this study was too small. The purpose of the research was to use ultrasound contrast agent microbubbles to locate the SLN only. This study enrolled the patients with negative nodes images, who were most likely to benefit from SLNB and avoid axillary lymph node dissection. In addition, the patients with a history of chemotherapy, radiotherapy, and axillary surgery were excluded, which inevitably resulted in selective bias. Secondly, due to the small sample size and only 12 lymph nodes were confirmed to be metastatic, the enhancement patterns of benign and malignant lymph nodes were not classified and summarized. Some investigations looked at patterns of lymph node enhancement, and it showed that unenhanced sentinel nodes associated with discontinuous lymphatic veins were the most likely to be positive $(10,16,17)$. Thirdly, in this paper, most of the SLN were pathologically negative lymph nodes with loose and soft textures. Although not present in this study, the risk of slippage of the metal wire should be aware. Breast tissue marker clips combing the radiograph of the specimen are expected to further improve the stability of the procedure. More cases and multi center studies are necessary.

In summary, intradermal injection of an ultrasound contrast agent in the areola area was a convenient and effective procedure for identifying and localizing SLNs. Compared with the traditional techniques, the CEUS method was an effective supplement with the advantage of safety, better accuracy, slight trauma, short operative time, and fewer postoperative complications. Larger sample sizes and multicenter studies are necessary to determine whether this new technique be used routinely for identified SLNs for early breast cancer.

\section{Acknowledgments}

Funding: The work was supported by the Chinese National Key Research and Development Project (No. 2018YFC1315601) and the Tianjin health research project (No. KJ20024).

\section{Footnote}

Reporting Checklist: The authors have completed the STARD reporting checklist. Available at https://gs.amegroups.com/ 
article/view/10.21037/gs-22-10/rc

Data Sharing Statement: Available at https://gs.amegroups. com/article/view/10.21037/gs-22-10/dss

Conflicts of Interest: All authors have completed the ICMJE uniform disclosure form (available at https://gs.amegroups. com/article/view/10.21037/gs-22-10/coif). The authors have no conflicts of interest to declare.

Ethical Statement: The authors are accountable for all aspects of the work in ensuring that questions related to the accuracy or integrity of any part of the work are appropriately investigated and resolved. All procedures performed in this study were in accordance with the Declaration of Helsinki (as revised in 2013). The study was approved by Institutional Review Board of Tianjin Medical University Cancer Institute and Hospital (No. bc2018015). Individual consent for this retrospective analysis was waived.

Open Access Statement: This is an Open Access article distributed in accordance with the Creative Commons Attribution-NonCommercial-NoDerivs 4.0 International License (CC BY-NC-ND 4.0), which permits the noncommercial replication and distribution of the article with the strict proviso that no changes or edits are made and the original work is properly cited (including links to both the formal publication through the relevant DOI and the license). See: https://creativecommons.org/licenses/by-nc-nd/4.0/.

\section{References}

1. Sung H, Ferlay J, Siegel RL, et al. Global Cancer Statistics 2020: GLOBOCAN Estimates of Incidence and Mortality Worldwide for 36 Cancers in 185 Countries. CA Cancer J Clin 2021;71:209-49.

2. Peristeri DV, Harissis HV. Axillary lymph node dissection vs sentinel biopsy only among women with early-stage breast cancer and sentinel node metastasis: A systematic review and meta-analysis. Breast J 2021;27:158-64.

3. Giuliano AE, Ballman KV, McCall L, et al. Effect of Axillary Dissection vs No Axillary Dissection on 10-Year Overall Survival Among Women With Invasive Breast Cancer and Sentinel Node Metastasis: The ACOSOG Z0011 (Alliance) Randomized Clinical Trial. JAMA 2017;318:918-26.

4. Donker M, van Tienhoven G, Straver ME, et al. Radiotherapy or surgery of the axilla after a positive sentinel node in breast cancer (EORTC 10981-22023 AMAROS): a randomised, multi-centre, open-label, phase 3 non-inferiority trial. Lancet Oncol 2014;15:1303-10.

5. Sávolt Á, Péley G, Polgár C, et al. Eight-year follow up result of the OTOASOR trial: The Optimal Treatment Of the Axilla - Surgery Or Radiotherapy after positive sentinel lymph node biopsy in early-stage breast cancer: A randomized, single centre, phase III, non-inferiority trial. Eur J Surg Oncol 2017;43:672-9.

6. Solá M, Alberro JA, Fraile M, et al. Complete axillary lymph node dissection versus clinical follow-up in breast cancer patients with sentinel node micrometastasis: final results from the multicenter clinical trial AATRM 048/13/2000. Ann Surg Oncol 2013;20:120-7.

7. Galimberti V, Cole BF, Viale G, et al. Axillary dissection versus no axillary dissection in patients with breast cancer and sentinel-node micrometastases (IBCSG 23-01): 10year follow-up of a randomised, controlled phase 3 trial. Lancet Oncol 2018;19:1385-93.

8. Sever AR, Mills P, Jones SE, et al. Preoperative sentinel node identification with ultrasound using microbubbles in patients with breast cancer. AJR Am J Roentgenol 2011;196:251-6.

9. Breast Cancer Expert Committee of National Cancer Quality Control Center; Breast Cancer Expert Committee of China Anti-Cancer Association; Cancer Drug Clinical Research Committee of China Anti-Cancer Association. Guidelines for clinical diagnosis and treatment of advanced breast cancer in China (2020 Edition). Zhonghua Zhong Liu Za Zhi 2020;42:781-97.

10. Cui Q, Dai L, Li J, et al. Accuracy of CEUS-guided sentinel lymph node biopsy in early-stage breast cancer: a study review and meta-analysis. World J Surg Oncol 2020;18:112.

11. Veronesi U, Paganelli G, Galimberti V, et al. Sentinel-node biopsy to avoid axillary dissection in breast cancer with clinically negative lymph-nodes. Lancet 1997;349:1864-7.

12. Goldfarb LR, Alazraki NP, Eshima D, et al. Lymphoscintigraphic identification of sentinel lymph nodes: clinical evaluation of 0.22 -micron filtration of Tc99m sulfur colloid. Radiology 1998;208:505-9.

13. Alvarez S, Añorbe E, Alcorta P, et al. Role of sonography in the diagnosis of axillary lymph node metastases in breast cancer: a systematic review. AJR Am J Roentgenol 2006;186:1342-8.

14. Goldberg BB, Merton DA, Liu JB, et al. Sentinel lymph nodes in a swine model with melanoma: contrast-enhanced lymphatic US. Radiology 2004;230:727-34. 
15. Omoto K, Hozumi Y, Omoto Y, et al. Sentinel node detection in breast cancer using contrast-enhanced sonography with $25 \%$ albumin--Initial clinical experience. J Clin Ultrasound 2006;34:317-26.

16. Xie F, Zhang D, Cheng L, et al. Intradermal microbubbles and contrast-enhanced ultrasound (CEUS) is a feasible approach for sentinel lymph node identification in earlystage breast cancer. World J Surg Oncol 2015;13:319.

17. Barentsz MW, Verkooijen HM, Pijnappel RM, et al. Sentinel lymph node localization with contrast-enhanced ultrasound and an I-125 seed: an ideal prospective development study. Int J Surg 2015;14:1-6.

18. Li J, Lu M, Cheng X, et al. How Pre-operative Sentinel Lymph Node Contrast-Enhanced Ultrasound Helps Intraoperative Sentinel Lymph Node Biopsy in Breast Cancer: Initial Experience. Ultrasound Med Biol 2019;45:1865-73.

19. Shimazu K, Miyake T, Tanei T, et al. Real-Time Visualization of Lymphatic Flow to Sentinel Lymph Nodes by Contrast-Enhanced Ultrasonography with Sonazoid in Patients with Breast Cancer. Ultrasound Med Biol 2019;45:2634-40.

20. Shimazu K, Ito T, Uji K, et al. Identification of sentinel lymph nodes by contrast-enhanced ultrasonography with Sonazoid in patients with breast cancer: a feasibility study in three hospitals. Cancer Med 2017;6:1915-22.

21. Agliata G, Valeri G, Argalia G, et al. Role of ContrastEnhanced Sonography in the Evaluation of Axillary

Cite this article as: Xu YL, Liu XJ, Zhu Y, Lu H. Preoperative localization of sentinel lymph nodes using percutaneous contrast-enhanced ultrasonography in patients with breast cancer. Gland Surg 2022;11(2):369-377. doi: 10.21037/gs-22-10
Lymph Nodes in Breast Carcinoma: A Monocentric Study. J Ultrasound Med 2017;36:505-11.

22. Liu J, Liu X, He J, et al. Percutaneous contrast-enhanced ultrasound for localization and diagnosis of sentinel lymph node in early breast cancer. Sci Rep 2019;9:13545.

23. Wang Y, Zhou W, Li C, et al. Variation of sentinel lymphatic channels (SLCs) and sentinel lymph nodes (SLNs) assessed by contrast-enhanced ultrasound (CEUS) in breast cancer patients. World J Surg Oncol 2017;15:127.

24. Chagpar AB, Scoggins CR, Martin RC 2nd, et al. Prediction of sentinel lymph node-only disease in women with invasive breast cancer. Am J Surg 2006;192:882-7.

25. Piscaglia F, Bolondi L; Italian Society for Ultrasound in Medicine and Biology (SIUMB) Study Group on Ultrasound Contrast Agents. The safety of Sonovue in abdominal applications: retrospective analysis of 23188 investigations. Ultrasound Med Biol 2006;32:1369-75.

26. Yang S, Tang K, Tao J, et al. Clinical study of different parts of ultrasound contrast agent in the diagnosis of sentinel lymph node in breast cancer. Journal of Clinical and Experimental Medicine 2016;15:1773-6.

27. Sun Y, Mi C. Comparative Study of Intravenous and Percutaneous Contrast-enhanced Ultrasound in Detection of Sentinel Lymph Nodes in Breast Cancer. Journal of Ningxia Medical University 2012;34:480-2.

(English Language Editor: C. Gourlay) 